\title{
Multicenter Evaluation of a Fully Automated High- Throughput SARS-CoV-2 Antigen Immunoassay
}

\author{
Dominik Nörz · Flaminia Olearo · Stojan Perisic · Matthias F. Bauer • \\ Elena Riester · Tanja Schneider · Kathrin Schönfeld • Tina Laengin • \\ Marc Lütgehetmann (D)
}

Received: June 14, 2021 / Accepted: July 21, 2021 / Published online: August 9, 2021

(C) The Author(s) 2021

\begin{abstract}
Introduction: Molecular testing for severe acute respiratory syndrome coronavirus 2 (SARS-CoV-2) continues to suffer from delays and shortages. Antigen tests have recently emerged as a viable alternative to detect patients with high viral loads, associated with elevated risk of transmission. While rapid lateral flow tests greatly improved accessibility of
\end{abstract}

Dominik Nörz and Flaminia Olearo have contributed equally to this work.

Supplementary Information The online version contains supplementary material available at https:// doi.org/10.1007/s40121-021-00510-x.

D. Nörz · F. Olearo · M. Lütgehetmann ( $\varangle)$ Center for Diagnostics, Institute of Medical Microbiology, Virology and Hygiene, University Medical Center Hamburg-Eppendorf (UKE), Martinistraße 52, 20246 Hamburg, Germany e-mail: mluetgeh@uke.de

S. Perisic

Institute for Clinical Chemistry and Laboratory Medicine, Hospital of Stuttgart, Stuttgart, Germany

M. F. Bauer

Institute for Laboratory Medicine, Hospital of Ludwigshafen, Ludwigshafen, Germany

E. Riester

Labor Augsburg MVZ GmbH, Augsburg, Germany

T. Schneider · K. Schönfeld · T. Laengin

Roche Diagnostics GmbH, Penzberg, Germany
SARS-CoV-2 detection in critical areas, their manual nature limits scalability and suitability for large-scale testing schemes. The Elecsys ${ }^{\circledR}$ SARS-CoV-2 Antigen assay allows antigen immunoassays to be carried out on fully automated high-throughput serology platforms.

Methods: A total of 3139 nasopharyngeal and oropharyngeal swabs were collected at 3 different testing sites in Germany. Swab samples were pre-characterized by reverse transcription realtime polymerase chain reaction (RT-qPCR) and consecutively subjected to the antigen immunoassay on either the cobas e 411 or cobas e 801 analyzer.

Results: Of the tested respiratory samples, 392 were PCR positive for SARS-CoV-2 RNA. Median concentration was $2.95 \times 10^{4}$ (interquartile range [IQR] $5.1 \times 10^{2}-3.5 \times 10^{6}$ ) copies $/ \mathrm{ml}$. Overall sensitivity and specificity of the antigen immunoassay were $60.2 \%$ (95\% confidence interval [CI] 55.2-65.1) and 99.9\% (95\% CI 99.6-100.0), respectively. A $93.7 \%$ (95\% CI 89.7-96.5) sensitivity was achieved at a viral RNA concentration $\geq 10^{4}$ copies $/ \mathrm{ml} \quad(\sim$ cycle threshold $\left[C_{\mathrm{t}}\right]$ value $<29.9$ ).

Conclusion: The Elecsys SARS-CoV-2 Antigen assay reliably detected patient samples with viral loads $\geq 10,000$ copies $/ \mathrm{ml}$. It thus represents a viable high-throughput alternative for screening of patients or in situations where PCR testing is not readily available. 
Keywords: Antigen immunoassay; Highthroughput platform; SARS-CoV-2

\section{Key Summary Points}

\section{Why carry out this study?}

The SARS-CoV-2 pandemic has led to a surge in demand for reliable, mass diagnostic tests worldwide.

A thorough clinical evaluation of a fully automated high-throughput Elecsys ${ }^{\circledR}$ SARS-CoV-2 Antigen assay on a total of 3139 clinical samples pre-characterized by quantitative RT-PCR was carried out.

\section{What was learned from the study?}

The assay demonstrated excellent specificity (99.9\%) and good relative sensitivity, with an overall sensitivity of $60.2 \%$ and a sensitivity of $93.7 \%$ for samples containing $\geq 10^{4}$ viral RNA copies/ml.

The Elecsys SARS-CoV-2 Antigen assay is a viable high-throughput, automated alternative to manual lateral flow antigen tests.

\section{INTRODUCTION}

Reverse transcription real-time polymerase chain reaction (RT-qPCR) remains the gold standard for detection of severe acute respiratory syndrome coronavirus 2 (SARS-CoV-2) in clinical specimens due to its unparalleled analytic accuracy $[1,2]$. However, the sudden surge in demand for molecular testing continues to overstretch the capacity of both diagnostic laboratories and reagent suppliers, leading to reporting delays and sometimes inadequate availability of testing where it is urgently needed. SARS-CoV-2 antigen immunoassays have recently emerged as an alternative to nucleic acid amplification tests, most prolifically in the form of rapid, point-of-care, lateral flow tests
(LFTs) [3-5]. Despite their inherent disadvantage in sensitivity compared with PCR, mathematical modeling suggests that tests could be effective for infection control if appropriate testing frequency were adopted [6]. Using currently available rapid antigen tests for mass testing does however bring about additional challenges, as this assay format is highly manual in nature and largely unsuitable for automation. Moving the SARS-CoV-2 antigen assay from LFT to a high-throughput immunoanalyzer using electrochemiluminescence detection technology could improve those issues, while further increasing overall testing capacity. The aim of this study was to evaluate the sensitivity and specificity of the new fully automated Elecsys ${ }^{\circledR}$ SARS-CoV-2 Antigen assay, using samples characterized by the gold standard: RT-qPCR.

\section{METHODS}

\section{Samples}

In this multicenter study, nasopharyngeal and oropharyngeal samples were tested following routine diagnostics at the University Medical Center Hamburg-Eppendorf, the Hospital of Stuttgart, and Hospital Ludwigshafen (Supplementary Table 1). Overall, the study comprised a total of 3139 nasopharyngeal and oropharyngeal swab samples, including 1331 samples from the Hospital of Stuttgart, 1058 samples from University Medical Center Hamburg-Eppendorf, and 647 samples from Hospital Ludwigshafen as well as 103 banked nasopharyngeal samples in a universal viral transport (UVT) system from a commercial vendor (Boca Biolistics, Pompano Beach, FL, USA). Specimens were collected in November 2020 in $3 \mathrm{ml}$ Copan Universal Transport Medium (UTM-RT, Copan, Brescia, Italy) or BD Universal Viral Transport (UVT, Becton Dickinson, Sparks, MD, USA) using flocked swabs.

This study was conducted in accordance with applicable regulations, the study protocol provided by Roche Diagnostics, and the principles of the Declaration of Helsinki. The use of anonymized remnant samples material was 
approved by ethical review committees prior to study initiation (ethics committee names and approval numbers: Ethik-Kommission bei der Landesärztekammer Baden-Württemberg; F-2020-154 [Stuttgart]; Ethik-Kommission der Ärztekammer Hamburg: WF-184/20 [Hamburg]; Ethik-Kommission bei der Landesärztekammer Rheinland-Pfalz: 2020-15449 [Ludwigshafen]).

\section{Antigen Assay and RT-qPCR}

Antigen detection was performed at two testing sites (University Medical Center Hamburg-Eppendorf [UKE] and testing site of Augsburg) using the Elecsys SARS-CoV-2 Antigen assay on the cobas e 411 and cobas e 801 immunoanalyzers. The Elecsys SARS-CoV-2 Antigen assay (Roche Diagnostics International Ltd, Rotkreuz, Switzerland) is an automated electrochemiluminescence immunoassay (ECLIA), developed for in vitro qualitative detection of SARS-CoV-2 antigen. It utilizes monoclonal antibodies directed against the SARS-CoV-2 nucleocapsid protein in an antibody sandwich assay format for the detection of SARS-CoV-2 in upper respiratory tract specimens [7]. The time to result for the assay can be as little as $18 \mathrm{~min}$, and the analyzer automatically calculates a cutoff based on the measurement of two calibrators: one negative (COV2AG Cal1) and one positive (COV2AG Cal2). The results obtained are interpreted as recommended by the manufacturer as either 'reactive' or 'non-reactive' in the form of a cutoff index (COI), i.e., 'non-reactive' if $\mathrm{COI}<1.0$ or 'reactive' if COI $\geq 1.0$.

RT-qPCR testing was performed with the qualitative cobas $^{\circledR}$ SARS-CoV-2 assay on the cobas $^{\circledR} 6800$ system (Roche Molecular Systems, Inc., Branchburg, NJ, USA), according to the manufacturer's instructions [8]. Quantification was performed using cycle threshold $\left(C_{\mathrm{t}}\right)$ values of Target-2 (envelope protein coding gene) [9] using reference material by Qnostics Ltd. (Glasgow, UK) as the quantification standard. The formula for conversion of the $C_{\mathrm{t}}$ value to $\log 10$ copies $/ \mathrm{ml}$ is $\left(C_{\mathrm{t}} \times-0.30769\right)+13.2$. The lower limit of quantification was set to $C_{\mathrm{t}}=33$ corresponding to 1000 copies SARS-CoV-2 RNA/ $\mathrm{ml}$, in accordance with previous studies [9]. It has to be noted that the PCR test and quantification standard used for generating quantitative results were not recommended for this purpose by the respective manufacturers, and reliability is expected to be lower than commercial quantitative solutions once they become available, which represents a limitation of this study.

\section{Statistical Analysis}

Sensitivity and specificity, including 95\% confidence intervals (CIs), were assessed as per Altman [10]. Pearson correlation was used for linear regression. The significance threshold was set at a two-sided alpha value of 0.05 . Statistical analysis was performed with STATA (version 15) and GraphPad Prism (version 86 9.0.0).

\section{Data Availability}

Qualified researchers may request access to individual patient-level data through the clinical study data request platform (https://vivli. org/). Further details on Roche's criteria for eligible studies are available here: https://vivli.org/ members/ourmembers/. For further details on Roche's Global Policy on the Sharing of Clinical Information and how to request access to related clinical study documents, see: https://www. roche.com/research_and_development/who_ we_are_how_we_work/clinical_trials/our_commit ment_to_data_sharing.htm.

\section{RESULTS}

A total of 3139 respiratory samples were analyzed in this study (Supplementary Table 1). Three hundred ninety-two samples were SARSCoV-2 RNA positive and 2747 samples were SARS-CoV-2 RNA negative. For PCR-positive samples, 79/392 (20.2\%) patients were asymptomatic and $79.8 \%(313 / 392)$ were symptomatic, with $47.6 \%(149 / 313)$ of samples collected within the first 5 days of symptom onset. The median concentration of viral RNA was $2.95 \times 10^{4}$ SARS-CoV-2 copies/ml (interquartile range [IQR] $5.1 \times 10^{2}-3.5 \times 10^{6}$ SARS-CoV-2 RNA copies/ml; Fig. 1a). In total, 

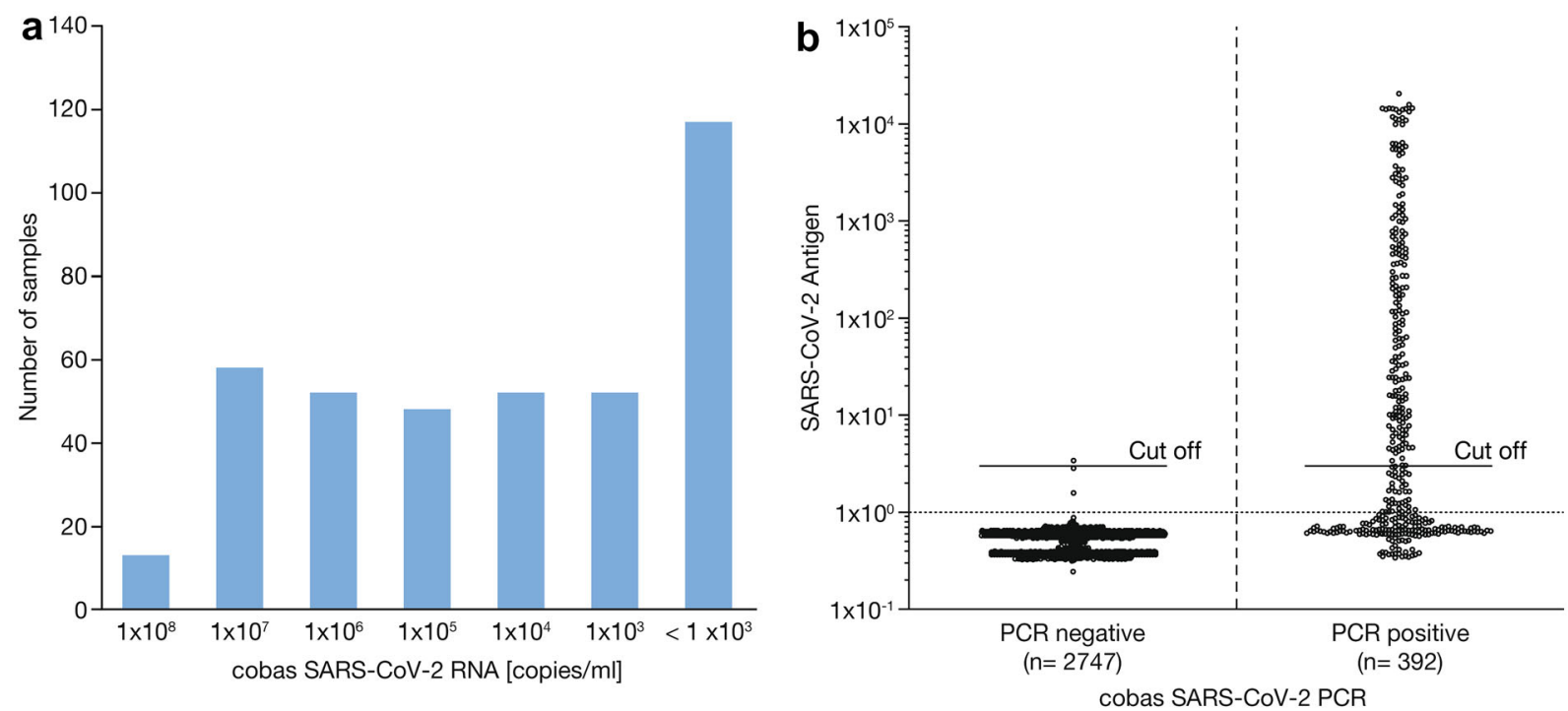

Fig. 1 a Number of positive samples relative to cobas SARS-CoV-2 PCR titer [copies/ml]; b COI values of Elecsys SARSCoV-2 Antigen test from 2747 cobas SARS-CoV-2 PCR-negative and 392 cobas SARS-CoV-2 PCR-positive samples

236/392 samples were detected as reactive for SARS-CoV-2 antigen with the Elecsys assay, with a median COI value of 46.0 (IQR 6.0-701.3). Distribution of the results for the PCR-positive and -negative groups are shown in Fig. 1b. The overall sensitivity and specificity were $60.2 \%$ (95\% CI 55.2-65.1, number of samples $[N]=392)$ and $99.9 \% \quad(95 \%$ CI $99.6-100.0$,
$N=2747$ ), respectively (Table 1 ). The cumulative sensitivity was $93.7 \%, 100.0 \%$, and $100.0 \%$ for samples with $>10,000$ copies $\left(\sim C_{\mathrm{t}}<29.9\right)$, 100,000 copies $\left(\sim C_{\mathrm{t}}<26.6\right)$, and $1,000,000$ copies $\left(\sim C_{\mathrm{t}}<23.0\right)$ (Table 1$)$, respectively.

For samples from symptomatic patients, the assay reached an overall relative sensitivity of $68.4 \%$ (95\% CI 62.9-73.5, $N=313)$, increasing

Table 1 Sensitivity and specificity results for the Elecsys SARS-CoV-2 Antigen assay

\begin{tabular}{|c|c|c|c|c|c|}
\hline & $\begin{array}{l}\text { cobas SARS- } \\
\text { CoV-2 assay }\end{array}$ & $N$ total & $\begin{array}{l}\text { Elecsys SARS-CoV-2 } \\
\text { Antigen negative }\end{array}$ & $\begin{array}{l}\text { Elecsys SARS-CoV- } \\
2 \text { Antigen positive }\end{array}$ & $\begin{array}{l}\text { Relative sensitivity/ } \\
\text { specificity, \% ( } 95 \% \text {, CI two- } \\
\text { sided) }\end{array}$ \\
\hline \multirow{4}{*}{$\begin{array}{l}\text { Relative } \\
\text { sensitivity }\end{array}$} & PCR positive & 392 & 156 & 236 & $60.2(55.2-65.1)$ \\
\hline & $\begin{array}{r}>10^{4} \text { copies } / \mathrm{ml} \\
\left(\sim C_{\mathrm{t}}<29.9\right)\end{array}$ & 223 & 14 & 209 & $93.7(89.7-96.5)$ \\
\hline & $\begin{array}{r}>10^{5} \text { copies } / \mathrm{ml} \\
\left(\sim C_{\mathrm{t}}<26.6\right)\end{array}$ & 171 & 0 & 171 & $100.0(97.9-100.0)$ \\
\hline & $\begin{array}{r}>10^{6} \text { copies } / \mathrm{ml} \\
\quad\left(\sim C_{\mathrm{t}}<23.0\right)\end{array}$ & 122 & 0 & 122 & $100.0(97.0-100.0)$ \\
\hline $\begin{array}{l}\text { Relative } \\
\text { specificity }\end{array}$ & PCR negative & $2747^{\mathrm{a}}$ & 2743 & 4 & $99.9(99.6-100.0)$ \\
\hline
\end{tabular}

Total positive and total negative are given in bold

$C I$ confidence interval, $C_{t}$ cycle threshold, $N$ number of samples

${ }^{a}$ Fifteen samples invalid with cobas SARS-CoV-2 RT-PCR, but negative with another SARS-CoV-2 RT-PCR test 
to $83.2 \%(95 \%$ CI $76.2-88.8, N=149)$ if symptoms had manifested within the last 5 days (Supplementary Table 2). On the other hand, the overall sensitivity observed in asymptomatic patients was only $27.8 \% \quad(95 \%$ CI 18.3-39.1, $N=79$ ). Relative sensitivities corresponded with high RNA levels and gradually decreased as RNA levels decreased. A considerable increase in sensitivity was observed when analysis was limited to samples containing $>10,000$ copies $/ \mathrm{ml}$, reaching 95.9\% (95\% CI $92.2-98.2, \quad N=197)$ for symptomatic patients and 76.9\% (95\% CI 56.4-91.0, $N=26$ ) for asymptomatic patients (Supplementary Table 2).

To analyze the overall assay performance, samples were sorted by SARS-CoV-2 RNA copies/ $\mathrm{ml}$ and individual sensitivities calculated for sets of 20 samples (shown using a heat map in Fig. 2a). Of note, the sensitivity of the assay was $95 \%$ and $50 \%$ at concentrations of around $2 \times 10^{5}$ and $2 \times 10^{4}-5 \times 10^{3}$ viral copies $/ \mathrm{ml}$, respectively, but $<10 \%$ for samples with $<10^{3}$ copies $/ \mathrm{ml}$. When the analysis was focused on samples containing RNA levels beyond 95\% sensitivity of the immunoassay $(10,000$ copies/ $\left.\mathrm{ml} ; C_{\mathrm{t}}<29.9\right)$, a significant linear correlation was observed between the Elecsys SARS-CoV-2 Antigen assay COI value and SARS-CoV-2 RNA copies/ml $\left(p<0.0001 ; r^{2}=0.786\right.$; Fig. $\left.2 b\right)$. Also, in this group, a sensitivity of $98.3 \%$ (95\% CI 94.0-99.8) was achieved in the sub-analysis of patients tested $<5$ days after symptom onset.

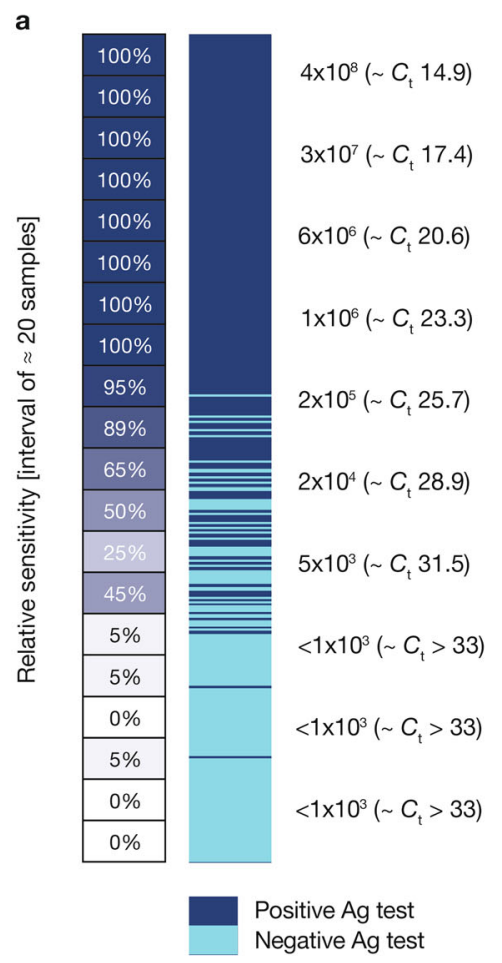

Fig. 2 a Elecsys SARS-CoV-2 Antigen assay results in relation to cobas SARS-CoV-2 RNA copies/ml. Dark blue fields represent positive and light blue fields represent negative Elecsys SARS-CoV-2 Antigen test results. On left side, sensitivity was calculated every $\approx 20$ samples included in the interval of the heatmap (copies $/ \mathrm{ml}$ ); b correlation between Elecsys SARS-CoV-2 Antigen assay $(\log 10[\mathrm{COI}], y$-axis) and cobas SARS-CoV-2 PCR assay

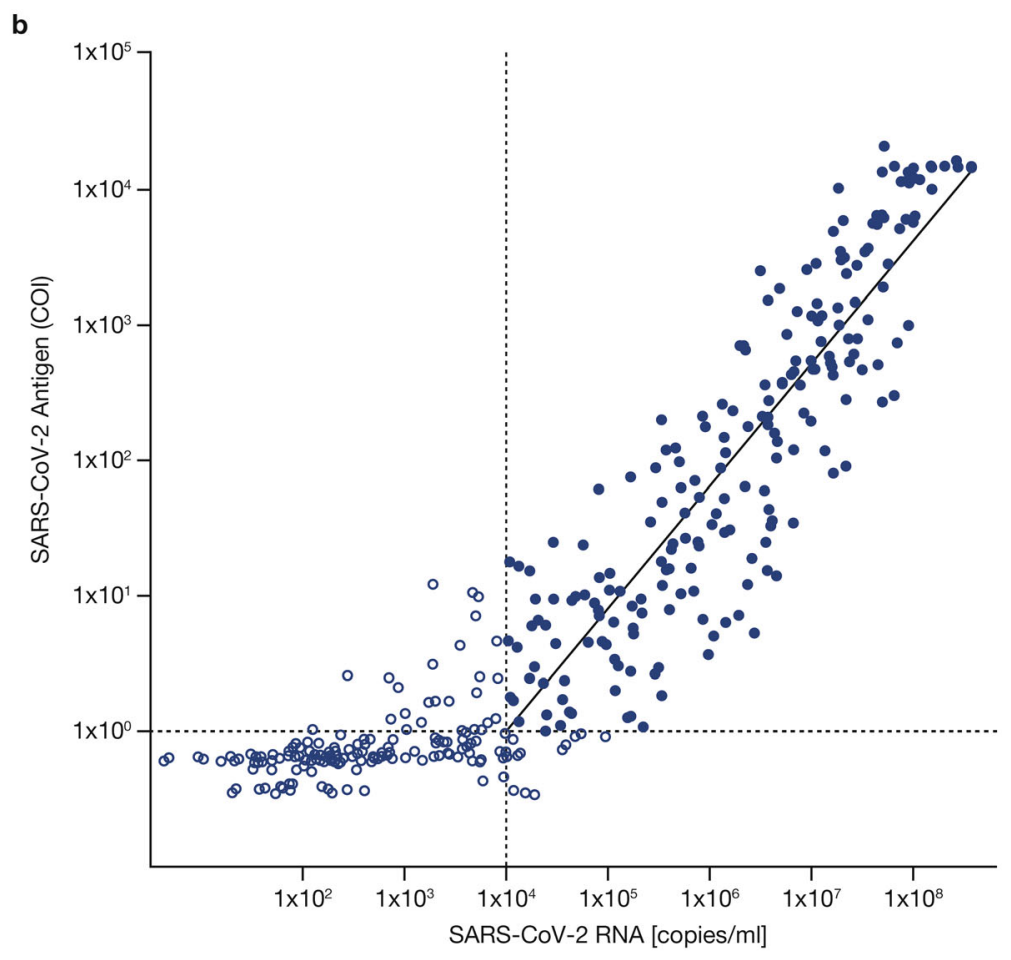

$(\log 10$ [RNA copies/ml], $\mathrm{x}$-axis). Closed blue circles: measurements within linear range considered for the trendline and correlation (SARS-CoV-2 RNA $\geq 10^{5}$ copies $/ \mathrm{ml}$; COI $\geq 1$ ). Open blue circles: measurements outside of the linear range of Elecsys SARS-CoV-2 or one cobas SARS-CoV-2 PCR Target negative, not considered for trendline and correlation. $p<0.0001 ; r^{2}=0.786 . \mathrm{Ag}$ antigen, $C O I$ cutoff index, $C_{t}$ cycle threshold 


\section{DISCUSSION}

The introduction of rapid SARS-CoV-2 antigen testing into the market was met with a lot of initial enthusiasm because of the promise of broad availability and easy application. While there are many different methods for antigen detection in clinical practice, such as enzymelinked immunosorbent assays and electrochemiluminescence immunoassays, the first wave of commercial tests consisted predominantly of rapid, point-of-care LFTs with optical read-outs. Preliminary performance data demonstrated good sensitivity in high-viralload samples, but low concentrations of virus are frequently missed, thus leading to the sobering conclusion that antigen tests cannot provide the same definitive results as RT-qPCR $[5,11-13]$. Although PCR tests are analytically superior, their effectiveness for infection control has often been hampered by supply shortages and reporting delays.

Recent studies have shown a correlation between the probability of recovering infectious virus from clinical samples and the time of onset of symptoms as well as RNA concentrations $\left(>1 \times 10^{6}\right.$ copies $\left./ \mathrm{ml}\right)$ within the specimen $[14,15]$. This suggests that the risk of transmission is highest during the early stages of disease, when viral titers are at their peak, which would also facilitate detection by antigen test. A recent study by McKay et al. [16] demonstrated good correlation between positive antigen test and positive viral culture; however, negative viral culture should not be misinterpreted as a reliable indicator for ruling out transmission risk. It has further been suggested that a low sensitivity test can be just as effective at detecting infections as a highly sensitive one, if testing can be made possible at increased frequency [6]. Most currently available SARS-CoV-2 antigen tests utilize a rapid, point-of-care LFT format, thus relying on a high degree of human interaction. They are unsuitable for automation and additional human operators are required at the same proportion as testing capacity is scaled up. While there are clear benefits of a point-of-care test that can be carried out with minimal training and requiring no specialized equipment [17], testing en masse (e.g., in healthcare facilities) is vastly more efficient when performed automatically using high-throughput immunoassay platforms.

In this study, we stratified samples to assess the relative sensitivity of the new Elecsys SARSCoV-2 Antigen assay for the cobas immunoassay analyzer systems using the gold standard, RT-qPCR. Although the overall relative sensitivity for the entire sample set was $60.2 \%$, among those patients that were tested in the first 5 days from the onset of symptoms, the sensitivity increased to $83.2 \%$. Furthermore, the Elecsys SARS-CoV-2 Antigen assay was able to detect $93.7 \%$ of patients with elevated risk of transmission $\left(C_{\mathrm{t}}<30\right.$ and accordingly RNA levels $>10,000$ copies $/ \mathrm{ml}$ ). Overall relative specificity was determined as $99.9 \%$ in a total of 2747 negative samples. These results are in line with existing preliminary data on the clinical performance of other available SARS-CoV-2 antigen tests $[12,18]$, including other highthroughput SARS-CoV-2 antigen tests like the Lumipulse $^{\circledR}$ [19], demonstrating a cumulative sensitivity of $55.2 \%$ compared with PCR.

The main limitation of the present study is that it is not a head-to-head comparison of the different systems on the market, and future investigation is needed to address this concern. Additionally, as antigen tests show the best performance in people with symptoms and within a certain number of days since symptom onset [3], the presence of symptoms should be considered when interpreting results from these assays. However, the high-throughput Elecsys SARS-CoV-2 Antigen assay might be a valuable analytic option in the coming months in the struggle to control the pandemic.

\section{CONCLUSIONS}

The novel Elecsys SARS-CoV-2 Antigen immunoassay showed good performance in a broad set of clinical samples compared to RTPCR. It demonstrated a high sensitivity (93.7\%) in detecting samples containing high concentrations of viral RNA ( $\geq 10^{4}$ viral RNA copies/ $\mathrm{ml})$, indicative of high transmission potential. Furthermore, a relative specificity of $99.9 \%$ 
ensures a minimal rate of false positives, consequently saving resources on confirmationtesting and unnecessary quarantine. The ability to run the test on high-throughput immunoassay platforms and a time-to-result of approximately $18 \mathrm{~min}$ allow for en masse deployment of SARS-CoV-2 antigen testing, for example, in the context of centralized largescale testing schemes.

\section{ACKNOWLEDGEMENTS}

Funding. This study was funded by Roche Diagnostics GmbH (Mannheim, Germany). Publication fees were funded by Roche Diagnostics International Ltd, Rotkreuz, Switzerland.

Medical Writing, Editorial, and Other Assistance. The authors thank: the serology team of the University Medical Center Hamburg-Eppendorf, Institute of Medical Microbiology, Virology and Hygiene; the laboratory team of the Institute of Laboratory Diagnostics, Hygiene and Transfusion Medicine at the Klinikum Ludwigshafen; the Corona specimen acquisition team and its Coordinator, Mr. OA Christian Menzel (Clinic of Stuttgart), Corona testing facility team (Clinic of Stuttgart), as well as Ms. Pauline Weissleder and Ms. Sina Semenowitsch (Clinic of Stuttgart) for their great help with specimen handling and data processing; Sigrid Reichhuber, Janina Edion, and Yvonne Knack (Roche Diagnostics) for management of investigation sites, data acquisition, and study monitoring. Medical writing support, under the direction of the authors, was provided by Tina Patrick (Elements Communications Ltd, Westerham, Kent, UK) and was funded by Roche Diagnostics International Ltd, Rotkreuz, Switzerland. COBAS, COBAS E and ELECSYS are trademarks of Roche. All other product names and trademarks are the property of their respective owners.

Authorship. All named authors meet the International Committee of Medical Journal Editors (ICMJE) criteria for authorship for this article, take responsibility for the integrity of the work as a whole, and have given their approval for this version to be published.

Authorship Contributions. Tina Laengin, Tanja Schneider, and Kathrin Schönfeld developed the study conceptualization and design and study protocol, and managed the study conduct, database generation and data validation. Marc Lütgehetmann, Stojan Perisic, and Matthias F. Bauer provided sample material and Marc Lütgehetmann, Stojan Perisic, and Elena Riester generated data during study testing. Marc Lütgehetmann, Tanja Schneider, and Tina Laengin conceived the original idea of the manuscript. Dominik Nörz, Flaminia Olearo, Marc Lütgehetmann, and Tanja Schneider formally analyzed the data and its representations for the manuscript. Dominik Nörz, Marc Lütgehetmann, and Flaminia Olearo drafted the manuscript and interpreted the data with critical input from Tanja Schneider and Tina Laengin. All authors reviewed and revised the manuscript and approved it for publication.

Disclosures. Tanja Schneider, Kathrin Schönfeld, and Tina Laengin are employees of Roche Diagnostics GmbH. Marc Lütgehetmann has received speaker's honoraria and related travel expenses from Roche Diagnostics. Elena Riester has received speaker's honorarium from Roche. Stojan Perisic, Flaminia Olearo, Matthias F. Bauer and Dominik Nörz have no conflicts to report.

Compliance with Ethics Guidelines. This study was conducted in accordance with applicable regulations, the study protocol provided by Roche Diagnostics, and the principles of the Declaration of Helsinki of 1964, and its later amendments. The use of anonymized remnant samples material was approved by ethical review committees prior to study initiation (ethics committee names and approval numbers: Ethik-Kommission bei der Landesärztekammer Baden-Württemberg; F-2020-154 [Stuttgart]; Ethik-Kommission der Ärztekammer Hamburg: WF-184/20 [Hamburg]; Ethik-Kommission bei der Landesärztekammer Rheinland-Pfalz: 2020-15449 [Ludwigshafen]). 
Data Availability. The datasets generated during and/or analyzed during the current study are available from the corresponding author on reasonable request.

Open Access. This article is licensed under a Creative Commons Attribution-NonCommercial 4.0 International License, which permits any non-commercial use, sharing, adaptation, distribution and reproduction in any medium or format, as long as you give appropriate credit to the original author(s) and the source, provide a link to the Creative Commons licence, and indicate if changes were made. The images or other third party material in this article are included in the article's Creative Commons licence, unless indicated otherwise in a credit line to the material. If material is not included in the article's Creative Commons licence and your intended use is not permitted by statutory regulation or exceeds the permitted use, you will need to obtain permission directly from the copyright holder. To view a copy of this licence, visit http:// creativecommons.org/licenses/by-nc/4.0/.

\section{REFERENCES}

1. World Health Organization. Laboratory testing for coronavirus disease 2019 (COVID-19) in suspected human cases. 2020. https://www.who.int/ publications-detail/laboratory-testing-for-2019-novelcoronavirus-in-suspected-human-cases-20200117. Accessed 9 July 2021.

2. Corman VM, Landt O, Kaiser M, et al. Detection of 2019 novel coronavirus (2019-nCoV) by real-time RT-PCR. Euro Surveill. 2020;25:2000045. https:// doi.org/10.2807/1560-7917.ES.2020.25.3.2000045.

3. Centers for Disease Control and Prevention. Interim Guidance for Antigen Testing for SARSCoV-2. 2021. https://www.cdc.gov/coronavirus/ 2019-ncov/lab/resources/antigen-tests-guidelines. html. Accessed 9 July 2021.

4. Iglòi Z, Velzing J, van Beek J, et al. Clinical evaluation of the Roche SD Biosensor rapid antigen test for SARS-CoV-2 in Municipal Health Service Testing Site, The Netherlands. Emerg Infect Dis. 2021;27: 1323-9. https://doi.org/10.3201/eid2705.204688.
5. Olearo F, Nörz D, Heinrich F, et al. Handling and accuracy of four rapid antigen tests for the diagnosis of SARS-CoV-2 compared to RT-qPCR. J Clin Virol. 2021;137:104782. https://doi.org/10.1016/j. jcv.2021.104782.

6. Mina MJ, Parker R, Larremore DB. Rethinking covid-19 test sensitivity-a strategy for containment. N Engl J Med. 2020;383:e120. https://doi. org/10.1056/NEJMp2025631.

7. Roche Diagnostics GmbH. Elecsys ${ }^{\circledR}$ SARS-CoV-2 Antigen method sheet v1. 2021. https://diagnostics. roche.com/global/en/products/params/elecsys-sarscov-2-antigen-test.html. Accessed 9 July 2021.

8. Roche Molecular Systems Inc. cobas ${ }^{\circledR}$ SARS-CoV-2 Qualitative assay for use on the cobas ${ }^{\circledR} 6800 / 8800$ Systems. 09179917001-07EN. Doc Rev. 7.0. https:// www.fda.gov/media/136049/download. Accessed 9 July 2021.

9. Nörz D, Frontzek A, Eigner U, et al. Pushing beyond specifications: evaluation of linearity and clinical performance of the cobas 6800/8800 SARS-CoV-2 RT-PCR assay for reliable quantification in blood and other materials outside recommendations. J Clin Virol. 2020;132:104650. https://doi.org/10. 1016/j.jcv.2020.104650.

10. Altman DG, Bland JM. Diagnostic tests. 1: sensitivity and specificity. BMJ. 1994;308:1552. https:// doi.org/10.1136/bmj.308.6943.1552.

11. Scohy A, Anantharajah A, Bodéus M, et al. Low performance of rapid antigen detection test as frontline testing for COVID-19 diagnosis. J Clin Virol. 2020;129:104455. https://doi.org/10.1016/j. jcv.2020.104455.

12. Corman VM, Haage VC, Bleicker T, et al. Comparison of seven commercial SARS-CoV-2 rapid pointof-care antigen tests. Lancet Microbe. 2021;2: e311-19. 5247(21)00056-2.

13. Krüger LJ, Gaeddert M, Tobian F, et al. Evaluation of the accuracy and ease-of-use of Abbott PanBio-a WHO emergency use listed, rapid, antigen-detecting point-of-care diagnostic test for SARS-CoV-2. PLoS ONE. 2021;16:e0247918. https://doi.org/10. 1371/journal.pone.0247918.

14. Jefferson T, Spencer EA, Brassey J, Heneghan C. Viral cultures for COVID-19 infectious potential assessment-a systematic review. Clin Infect Dis. 2020. https://doi.org/10.1093/cid/ciaa1764.

15. Woelfel R, Corman VM, Guggemos W, et al. Virological assessment of hospitalized patients with COVID-2019. Nature. 2020;581:465-9. https://doi. org/10.1038/s41586-020-2196-X. 
16. McKay SL, Tobolowsky FA, Moritz ED, et al. Performance evaluation of serial SARS-CoV-2 rapid antigen testing during a nursing home outbreak. Ann Intern Med. 2021;174:945-51. https://doi.org/ 10.7326/M21-0422.

17. Hoehl S, Schenk B, Rudych O, et al. At-home selftesting of teachers with a SARS-CoV-2 rapid antigen test to reduce potential transmissions in schools. medRxiv. 2020. https://doi.org/10.1101/2020.12. 04.20243410 .
18. Weitzel $\mathrm{T}$, Legarraga $\mathrm{P}$, Iruretagoyena $\mathrm{M}$, et al. Head-to-head comparison of four antigen-based rapid detection tests for the diagnosis of SARS-CoV2 in respiratory samples. bioRxiv. 2020. https://doi. org/10.1101/2020.05.27.119255.

19. Hirotsu Y, Maejima M, Shibusawa M, et al. Comparison of automated SARS-CoV-2 antigen test for COVID-19 infection with quantitative RT-PCR using 313 nasopharyngeal swabs, including from seven serially followed patients. Int J Infect Dis. 2020;99: 397-402. https://doi.org/10.1016/j.ijid.2020.08.029. 